\title{
ON THE STABILITY OF THE ABRAMOV TRANSFER FOR DIFFERENTIAL-ALGEBRAIC EQUATIONS OF INDEX 1*
}

\author{
THOMAS PETRY ${ }^{\dagger}$
}

\begin{abstract}
The transfer of boundary conditions for ordinary differential equations developed by Abramov [1] is a stable method for representing the solution spaces of linear boundary value problems. Instead of boundary value problems, matrix-valued initial value problems are solved. When integrating these differential equations, the inner independence of the columns of the solution matrix and, hence, of the solutions of the resulting linear system of equations, remains valid.

K. Balla and R. März have generalized Abramov's transfer for homogenized index 1 differentialalgebraic equations [6].

In this article, a direct version of the Abramov transfer for inhomogeneous linear index-1 differential-algebraic equations is developed and the numerical stability of this method is proved.
\end{abstract}

Key words. differential-algebraic equations, boundary value problems, transfer of boundary conditions, stability

AMS subject classifications. 65L10, 34B05

1. Introduction. Already since Lagrange [13], [2], [12] it has been known from the theory of ordinary regular differential equations that the solution space $M(t) \subset$ $\mathbb{R}^{m}$ of the initial value problem

$$
\begin{aligned}
y^{\prime}(t)+B(t) y(t) & =0 \\
C_{0} y\left(t_{0}\right) & =0
\end{aligned}
$$

can be described by the relation

$$
\phi^{T}(t) y(t)=\phi^{T}\left(t_{0}\right) y\left(t_{0}\right)=0
$$

if $\phi$ solves the IVP

$$
\begin{aligned}
\phi^{\prime}(t)-B^{T}(t) \phi(t) & =0, \\
\phi\left(t_{0}\right) & =C_{0}^{T}
\end{aligned}
$$

In case the $m \times k$-matrix $C_{0}$ has full row rank $k \leq m$, then the $k \times m$-matrix $\phi$ has full column rank $k$. The solution space $M(t)=\operatorname{ker} \phi^{T}(t)=(\operatorname{im} \phi(t))^{\perp}$ is now $(m-k)$-dimensional.

In general, it cannot be expected that the equation (2) is easier to integrate than (1). In particular, as in the numerical calculation of fundamental matrices, the columns of $\phi$, may become almost linearly dependent. Abramov [1] tried to find equations that are numerically easier to solve. By the orthogonalization ansatz $\phi^{T} \phi^{\prime} \equiv 0$ stable equations whose solutions provide a distinct equivalent description of the space $M(t)$ could be constructed. Although these equations are nonlinear, they have nice properties. First, they have a solution on the whole interval, which does not apply e.g. for the Riccati method in each case [2]. Second, they are stable. Moreover, the equations also provide $\phi^{T} \phi \equiv$ const., i.e., the columns of $\phi(t)$ remain

\footnotetext{
* This research was supported by German Scientific Research Fund, Grant Ma 1347/3-1

$\dagger$ Humboldt-Universität zu Berlin, Mathematisch-Naturwissenschaftliche Fakultät II, Institut für Angewandte Mathematik, D-10099 Berlin, Germany
} 
uniformly linearly independent, which is favourable for the numerical realization. It is not necessay to reorthogonalize as in the case of marching techniques or to change the imbedding as in the case of the Riccati method ([20], [4]) on account of numerically singular fundamental matrices. For the reader who is not familiar with Abramov's transfer method, an interesting, well known example of a regular ordinary differential equation where the Riccati method fails [4] is considered in section 6.1.

In section 2 fundamental notations are introduced and it is briefly shown in which way the solution spaces of differential-algebraic equations can be represented.

K. Balla and R. März [6] have extended Abramov's transfer to index 1 tractable differential-algebraic equations by means of homogenization. In this paper a new, direct approach to inhomogeneous linear DAEs is chosen (section 3). Two possible transformations of the adjoint DAE are described. The first possibility is the direct extension of Abramov's method to differential-algebraic equations, the second one corresponds to the approach by K. Balla and R. März [6]. The new approach described in section 3.1 allows an easier stability analysis (section 5) with a quantitative estimation of the error. Perturbations of the right-hand side can be interpreted as regular perturbations of the given problem. For the approach [6] such a result has not been known yet.

Numerical experiments are discussed in section 6 .

2. Representation of the Solution Spaces. We consider the index 1 tractable two-point boundary value problem

$$
\begin{aligned}
A(t) y^{\prime}(t)+B(t) y(t) & =f(t), \quad t_{0} \leq t \leq t_{1}, \\
C_{0} \cdot y\left(t_{0}\right) & =g_{0}, \\
C_{1} \cdot y\left(t_{1}\right) & =g_{1} .
\end{aligned}
$$

$A \in C^{1}\left(\left[t_{0}, t_{1}\right] ; \mathbb{R}^{m \times m}\right), B \in C\left(\left[t_{0}, t_{1}\right] ; \mathbb{R}^{m \times m}\right), f \in C\left(\left[t_{0}, t_{1}\right] ; \mathbb{R}^{m}\right)$.

Let $Q(t)$ be a projector function onto $N(t):=\operatorname{ker} A(t), P(t)=I-Q(t)$. Tractability with the index 1 is characterized by the fact that $\operatorname{rank} A(t)=$ const and the matrix $G(t):=A(t)+B(t) Q(t)$ is nonsingular for all $t \in\left[t_{0}, t_{1}\right]$ [16]. This notion is independent of the special choice of $Q$ [10, Theorem A.13], hence, one can choose $Q=I-A^{\dagger} A$, too. Under the above assumptions, $Q$ can be chosen as a $C^{1}$-function. Relations to other notions of index are presented in [16].

An important role for the index 1 tractable DAEs is played by $Q_{s}$, i.e., the canonical projector function onto the nullspace $N$ of $A$ along $S_{0}, S_{0}(t)=\left\{\xi \in \mathbb{R}^{m} \mid B(t) \xi \in\right.$ $\operatorname{im} A(t)\}$ and it holds that $Q_{s}(t)=Q(t) G^{-1}(t) B(t), P_{s}(t)=I-Q_{s}(t)[10$, p. 36]. In the case of index $1, S_{0}(t)$ is exactly the space of the solutions of the homogeneous problem (3a). Then

$$
S_{f}(t):=\left\{\xi \in \mathbb{R}^{m} \mid B(t) \xi-f(t) \in \operatorname{im} A(t)\right\}
$$

is the space of the solutions of the inhomogeneous problem (3a). Obviously, the condition $y(t) \in S_{f}(t)$ is equivalent to $Q_{s}(t) y(t)=Q(t) G^{-1}(t) f(t)$.

The adjoint problem of the given problem (3a) $[6]$ reads

$$
\left(A^{T} \phi\right)^{\prime}-B^{T} \phi=0
$$

or, equivalently due to the $C^{1}$-condition to $A$,

$$
A^{T} \phi^{\prime}-\left(B^{T}-A^{T^{\prime}}\right) \phi=0 .
$$


For each pair $y(t), \phi(t)$ of solutions of the equations (3a) and (4) it holds

$$
\left(\phi^{T}(t) A(t) y(t)\right)^{\prime}=\left(\phi^{T}(t) A(t)\right)^{\prime} y(t)+\phi^{T}(t) A(t) y^{\prime}(t)=\phi^{T}(t) f(t),
$$

and

$$
g^{\prime}(t)-\phi^{T}(t) f(t)=0,
$$

respectively, with the substitution

$$
g=\phi^{T} A y .
$$

Equation (5) has the explicit solution

$$
g(t)=\phi^{T}\left(t_{i}\right) A\left(t_{i}\right) y\left(t_{i}\right)+\int_{t_{i}}^{t} \phi^{T}(s) f(s) d t .
$$

Here, the index $i$ represents either 0 or 1 .

For the space defined by the equations (4)-(6) satisfying the boundary conditions (3b), (3c), the initial conditions for $\phi$ and $g$ in the differential equations (4), (5) have to be chosen appropriately. Due to equation (7) the relations $\phi^{T}\left(t_{i}\right) A\left(t_{i}\right)=D_{i} C_{i}$ and $g\left(t_{i}\right)=D_{i} g_{i}$ have to be valid for the initial values. $D_{i}$ is an arbitrary nonsingular matrix with an appropriate dimension.

Let $P_{\star s}(t)$ be the projector function along $N_{\star}(t):=\operatorname{ker} A^{T}(t)$ onto

$$
S_{\star}(t):=\left\{\xi \in R^{m}: \quad\left(B(t)^{T}-A^{T^{\prime}}(t)\right) \xi \in \operatorname{im} A^{T}(t)\right\} .
$$

If $C_{i} P\left(t_{i}\right)=C_{i}(i=0,1)$ holds, and we want to restrict ourselves to this case here, there are two simple possibilities for choosing the initial values of the system (4), (5), namely with $D_{i}=I$

$$
\begin{aligned}
& \phi\left(t_{i}\right)=P_{\star s}\left(t_{i}\right) \cdot A^{T \dagger}\left(t_{i}\right) \cdot C_{i}^{T}, \\
& g\left(t_{i}\right)=g_{i},
\end{aligned}
$$

and, on the other hand,

$$
\begin{aligned}
& \phi\left(t_{i}\right)=P_{\star s}\left(t_{i}\right) \cdot A^{T \dagger}\left(t_{i}\right) \cdot C_{i}^{T} L_{i}, \\
& g\left(t_{i}\right)=L_{i}^{T} g_{i},
\end{aligned}
$$

where $L_{i}$ is nonsingular, $L_{i} L_{i}^{T}=\left(C_{i} C_{i}^{T}\right)^{-1}$, which corresponds to the choice of $D_{i}=$ $L_{i}^{T}$.

The advantage of the second possibility presented here will be shown in section 3 .

We denote the solutions of the equations (4), (5) by $\phi_{i}, g_{i}$ for $i=0,1$ corresponding to the initial conditions (8a), (8b) and (8c), (8d), respectively.

The solution space of the initial value problem

$$
\begin{aligned}
A(t) y^{\prime}+B(t) y & =f(t), \quad t_{0} \leq t \leq t_{1}, \\
C_{i} \cdot y\left(t_{i}\right) & =g_{i}
\end{aligned}
$$


is given by the equations

$$
\begin{aligned}
\phi_{i}^{T}(t) A(t) y(t) & =g_{i}(t), \\
Q_{s}(t) y(t) & =Q(t) G^{-1}(t) f(t), \quad t \in\left[t_{0}, t_{1}\right],
\end{aligned}
$$

[6, Theorem 2.1], [5, THEOREM], $\phi_{i}$ and $g_{i}$ are solutions of the equations (4), (5) with the initial conditions (8a), (8b) and (8c), (8d), respectively.

We obtain the solution space of the boundary value problem (3a)-(3c) as the intersection of the solution spaces for $i=0,1$ given by the equations (9a), (9b).

REMARK 2.1. The above representation of the solution space is a generalization of the case of the ordinary regular differential equations, where $A \equiv I$ and equation (9b) is trivial because of $Q_{s}=Q \equiv 0$.

The function $\phi$ in equation $\left(4^{\star}\right)$ is matrix-valued. When solving the initial value problem $\left(4^{\star}\right),(8 \mathrm{a})$ resp. (8c) numerically, the columns of the matrix $A^{T} \phi$ may become almost linearly dependent. This unpleasant phenomenon can be prevented by means of an orthogonalization process.

3. Transformation of the Adjoint Equation. In this section we introduce transformations of the inhomogeneous system (4), (5), which go back to Abramov for the case of regular ordinary differential equations (e.g. [2], [1], [3]).

The following approach is chosen:

$$
\psi=\phi \cdot T, h=T^{T} g,
$$

with a nonsingular matrix function $T \in C^{1}\left(\left[t_{0}, t_{1}\right] ; \mathbb{R}^{m \times m}\right)$. Trivially, the resulting system

$$
\psi^{T}(t) A(t) y(t)=h(t)
$$

is equivalent to (9a), i.e., it can be taken instead of (9a) together with (9b).

Later, we want to choose the transformation (10) in such a way that it causes the desired orthogonalization effect.

Since $\psi T^{-1}=\phi$ satisfies the linear equations (4), (5),

$$
\begin{array}{r}
\left(A^{T} \psi\right)^{\prime}-B^{T} \psi-A^{T} \psi \cdot w=0, \\
h^{\prime}=w^{T} h+\psi^{T} f
\end{array}
$$

with $w:=T^{-1} T^{\prime}$ has to hold, too. This system of equations has index 1 .

The initial values (8a), (8b) resp. (8c), (8d) with $\psi$ and $h$ instead of $\phi$ and $g$ remain valid for the transformed system because the transformation, i.e., a multiplication of equation (6) from the left by $T^{T}$, does not change the solution spaces.

Let us now consider two possible transformations $T$. The first one is a generalization of Abramov's transfer [1], [2]. The second transformation is the Abramov transfer applied to the homogenized index 1 differential-algebraic equation (3a). It results in the equations obtained by K. Balla and R. März via homogenization [6].

3.1. First Transformation. We try to determine $T$ in such a way that

$$
\psi^{T} A\left(A^{T} \psi\right)^{\prime}=0
$$

holds. 
By this approach, equation (11) implies for $w$ the condition

$$
w=-\left(\psi^{T} A A^{T} \psi\right)^{-1} \psi^{T} A B^{T} \psi .
$$

Inserting $w$ into the equations (11) and (12) provides the transfer equations

$$
\begin{array}{r}
\left(A^{T} \psi\right)^{\prime}-\left(I-A^{T} \psi\left(\psi^{T} A A^{T} \psi\right)^{-1} \psi^{T} A\right) B^{T} \psi=0 \\
h^{\prime}+\psi^{T} B A^{T} \psi\left(\psi^{T} A A^{T} \psi\right)^{-1} h-\psi^{T} f=0 .
\end{array}
$$

Multiplying the differential equation (14a) by $\psi^{T} A$, transposing the result $\psi^{T} A\left(A^{T} \psi\right)^{\prime}=0$ and adding it to itself shows us that equation (14a) preserves the linear independence of the columns of $A^{T} \psi$, i.e., it holds that $\psi^{T} A A^{T} \psi=$ const. Thus, we have obtained an - in this sense - well-posed nonlinear transfer equation generalizing Abramov's approach from regular differential equations to differential-algebraic equations with index 1 immediately.

Hence, the property (13) inherent to the solutions of equation (14a) leads to $\psi^{T} A A^{T} \psi=C_{i}^{T} C_{i}$ for the first choice of the initial values (8a), (8b).

From the numerical point of view, the second choice of the initial values (8c), (8d) is of interest since then $\psi^{T} A A^{T} \psi=I$. Evaluating the equations (14a), (14b) a system containing the unit matrix as the coefficient matrix has to be solved. The columns of the product $A^{T} \psi$ remain orthogonal.

The differential equation for $h(14 \mathrm{~b})$ is completely decoupled from the equation for $\psi(14 \mathrm{a})$ in the sense that it can be solved separately in case there exists a solution $\psi$ of (14a).

The existence of a solution $\psi$ of equation (14a) on the whole interval $\left[t_{0}, t_{1}\right]$ was proved in [6, Theorem 2.1]. The existence of a solution $h$ of (14b) follows from the fact that $\psi$ exists as a solution of equation (14a) and thus equation (14b) is a linear differential equation w.r.t. $h$.

3.2. Second Transformation. Now $T$ is chosen such that

$$
\psi^{T} A\left(A^{T} \psi\right)^{\prime}+h h^{T^{\prime}}=0 .
$$

holds. This implies $W:=\psi^{T} A A^{T} \psi+h h^{T}=$ const. In a solution of equation (11) this leads to

$$
w=-W^{-1}\left(\psi^{T} A B^{T} \psi-h f^{T} \psi\right) .
$$

In this case the transfer equations read

$$
\begin{array}{r}
\left(A^{T} \psi\right)^{\prime}-\left(I-A^{T} \psi W^{-1} \psi^{T} A\right) B^{T} \psi+A^{T} \psi W^{-1} h f^{T} \psi=0, \\
h^{\prime}+\psi^{T} B A^{T} \psi W^{-1} h-\left(1-h^{T} W^{-1} h\right) \psi^{T} f=0 .
\end{array}
$$

Condition (15) is due to the endeavour for orthogonalization effects for the homogenized form with "enlarged" matrices (cf. [6]). The two equations (16a) and (16b) are coupled via the matrix $W . L_{i}$ is chosen in such a way that $L_{i} L_{i}^{T}=\left(C_{i} C_{i}^{T}+g_{i} g_{i}^{T}\right)^{-1}$. This implies $W=I$.

The existence of the solutions of the transfer equations (16a), (16b) on the whole interval $\left[t_{0}, t_{1}\right]$ was proved for an equivalent formulation in [6]. 
4. Algorithm. Now we can formulate a new algorithm for the transfer of the boundary condition for differential-algebraic equations:

1. Solve the equations (14a), (14b) with the corresponding initial conditions $(8 \mathrm{a}),(8 \mathrm{~b})$ or $(8 \mathrm{c}),(8 \mathrm{~d})$ on the left resp. right boundary of the interval, store the solution values.

2. Solve the algebraic equations

$$
\begin{aligned}
\psi_{i}^{T}(t) A(t) y(t) & =h_{i}(t), & i=0,1, \\
Q_{s}(t) y(t) & =Q(t) G^{-1}(t) f(t), & t \in\left[t_{0}, t_{1}\right],
\end{aligned}
$$

wherever the solution is needed.

REMARK 4.1. This algorithm is appropriate for multi-point boundary problems, too.

REMARK 4.2. For the basic problems, that is, the solution of the differential and algebraic equations, standard solution methods can be applied.

REMARK 4.3. Numerical methods preserving quadratic invariants (e.g. [8]), can be applied for solving the transfer equations.

REMARK 4.4. For well-posed boundary value problems these linear systems are uniquely solvable.

REMARK 4.5. For semiexplicit systems, the most frequently investigated ones in the literature, (e.g. ["7]),

$$
\begin{aligned}
u^{\prime}+B_{11} u+B_{12} v & =f_{1}, \\
B_{21} u+B_{22} v & =f_{2}
\end{aligned}
$$

the transfer equations become simpler because of the structure.

Let $\phi$ be partitioned as $\left(\phi_{1}^{T} \mid \phi_{2}^{T}\right)^{T}$. Then the transfer equations (14a), (14b) of the first variant of the transformation read

$$
\begin{aligned}
\phi_{1}^{\prime}-\left(I-\phi_{1}\left(\phi_{1}^{T} \phi_{1}\right)^{-1} \phi_{1}^{T}\right)\left(B_{11}^{T} \phi_{1}+B_{21}^{T} \phi_{2}\right) & =0, \\
B_{12}^{T} \phi_{1}+B_{22}^{T} \phi_{2} & =0, \\
h^{\prime}+\left(\phi_{1}^{T} B_{11}+\phi_{2}^{T} B_{21}\right) \phi_{1}\left(\phi_{1}^{T} \phi_{1}\right)^{-1} h-\left(\phi_{1}^{T} f_{1}+\phi_{2}^{T} f_{2}\right) & =0 .
\end{aligned}
$$

It is immediately obvious that this corresponds exactly to the classical Abramovtransfer for the inherent regular differential equation. However, in case of more complex structures, this does not apply any longer in general as shown by an example in [6].

The algebraic equations reduce to

$$
\begin{aligned}
\phi_{1,0}^{T} u & =h_{0}, \\
\phi_{1,1}^{T} u & =h_{1}, \\
B_{21} u+B_{22} v & =f_{2} .
\end{aligned}
$$

An analogous consideration with the transformation (15) provides similar equations.

REMARK 4.6. If one tries to solve nonlinear boundary value problems by means of quasilinearization, and if one applies the transfer method for solving the resulting systems of linear differential equations, then a modified Newton step is highly appropriate since only equation (14b) has to be solved in the first step of algorithm 4. Due to the coupling of the equations this does not hold for the second transformation. 
5. Backward Analysis. Now we investigate the stability of our algorithm (§ 4) relative to perturbations of the right-hand side of the transfer equations. This is necessary because, instead of the exact solutions $\psi, h$, only their approximations $\tilde{\psi}$, $\tilde{h}$ are available. In fact, instead of $(17 \mathrm{a}),(17 \mathrm{~b})$ only perturbed equations

$$
\begin{aligned}
\tilde{\psi}_{i}^{T}(t) A(t) \tilde{y}(t) & =\tilde{h}_{i}(t), & i=0,1, \\
Q_{s}(t) \tilde{y}(t) & =Q(t) G^{-1}(t) f(t), & t \in\left[t_{0}, t_{1}\right],
\end{aligned}
$$

are solved.

We perform the stability analysis for the equations (14a), (14b).

We consider functions $\tilde{\psi}$ and $\tilde{h}$ in a small neighbourhood of the soulution $\psi, h$. As these functions cannot be expected to fulfil the equations (14a), (14b) exactly, they are interpreted as solutions of the system

$$
\begin{aligned}
&\left(A^{T} \tilde{\psi}\right)^{\prime}-\left(I-A^{T} \tilde{\psi}\left(\tilde{\psi}^{T} A A^{T} \tilde{\psi}\right)^{-1} \tilde{\psi}^{T} A\right) B^{T} \tilde{\psi}=\varepsilon, \\
& \tilde{h}^{\prime}+\tilde{\psi}^{T} B A^{T} \tilde{\psi}\left(\tilde{\psi}^{T} A A^{T} \tilde{\psi}\right)^{-1} \tilde{h}-\tilde{\psi}^{T} f=\delta,
\end{aligned}
$$

hence, as solutions of the equations (14a), (14b) with perturbed right-hand side.

This method is denoted as "backward-analysis" in the literature (e.g. [19]).

In the following we aim at transforming the perturbed system (19a), (19b) into equations of the form (14a), (14b) with altered matrices $B$ and altered functions $f$. It turns out that the following procedure provides the desired result.

$$
\tilde{\psi}=\Psi K, \quad \tilde{h}=K^{T} \rho .
$$

Inserting the transformation (20) into the equations (19a), (19b) yields

$$
\begin{gathered}
\left(A^{T} \Psi\right)^{\prime} K+A^{T} \Psi K^{\prime}-\left(I-A^{T} \Psi\left(\Psi^{T} A A^{T} \Psi\right)^{-1} \Psi^{T} A\right) B^{T} \Psi K=\varepsilon \\
K^{T} \rho^{\prime}+K^{T^{\prime}} \rho+K^{T} \Psi^{T} B A^{T} \Psi\left(\Psi^{T} A A^{T} \Psi\right)^{-1} \rho-K^{T} \Psi^{T} f=\delta .
\end{gathered}
$$

If $K$ is chosen such that

$$
\Psi^{T} A\left(A^{T} \Psi\right)^{\prime}=0
$$

holds, then multiplying equation (21) from the left by $\Psi^{T} A$ and, subsequently, by $\left(\Psi^{T} A A^{T} \Psi\right)^{-1}$ provides the equation

$$
K^{\prime}=\left(\Psi^{T} A A^{T} \Psi\right)^{-1} \Psi^{T} A \varepsilon,
$$

which we insert into equation (21) then. After some transformations we obtain

$$
\left(A^{T} \Psi\right)^{\prime}-\left(I-A^{T} \Psi\left(\Psi^{T} A A^{T} \Psi\right)^{-1} \Psi^{T} A\right)\left(\varepsilon K^{-1}+B^{T} \Psi\right)=0 .
$$

From (20) we conclude the explicit representation of the inverse function of $K$.

$$
K^{-1}=\left(\tilde{\psi}^{T} A A^{T} \tilde{\psi}\right)^{-1} \tilde{\psi}^{T} A A^{T} \Psi
$$

Thus, the equations (19a), (19b) are transferred into the system

$$
\begin{array}{r}
\left(A^{T} \Psi\right)^{\prime}-\left(I-A^{T} \Psi\left(\Psi^{T} A A^{T} \Psi\right)^{-1} \Psi^{T} A\right) \tilde{B}^{T} \Psi=0 \\
\rho^{\prime}+\Psi^{T} \tilde{B} A^{T} \Psi\left(\Psi^{T} A A^{T} \Psi\right)^{-1} \rho-\Psi^{T} \tilde{f}=0 .
\end{array}
$$


$\tilde{B}, \tilde{f}$ are defined as follows

$$
\begin{gathered}
\tilde{B}=B+A A^{T} \tilde{\psi}\left(\tilde{\psi}^{T} A A^{T} \tilde{\psi}\right)^{-1} \varepsilon^{T} \\
\tilde{f}=f+A A^{T} \tilde{\psi}\left(\tilde{\psi}^{T} A A^{T} \tilde{\psi}\right)^{-1} \delta
\end{gathered}
$$

The equations (22a), (22b) are the transfer equations of the DAE

$$
A \tilde{y}^{\prime}+\tilde{B} \tilde{y}=\tilde{f}
$$

which is tractable with index 1 . This is immediately obvious because the leading coefficient $A$ from (3a) occurs here again, and also the relevant subspaces $S_{0}(t)$ to (3a) and $\tilde{S}_{0}(t):=\left\{\xi \in \mathbb{R}^{m} \mid \tilde{B}(t) \xi \in \operatorname{im} A(t)\right\}$ coincide because of

$$
\begin{aligned}
\tilde{S}_{0}(t) & =\left\{\xi \in R^{m}: B(t) \xi+A(t) A^{T} \tilde{\psi}\left(\tilde{\psi}^{T} A A^{T} \tilde{\psi}\right)^{-1} \varepsilon^{T} \xi \in \operatorname{im} A(t)\right\} \\
& =\left\{\xi \in R^{m}: B(t) \xi \in \operatorname{im} A(t)\right\} \\
& =S_{0}(t) .
\end{aligned}
$$

As a transfer equation of an index 1 DAE, (22a), (22b) has solutions, which justifies our approach on principle.

Well, how to characterize the error $\tilde{y}(t)-y(t)$ more exactly? We will give an answer to this question in the following theorem.

TheOREM 5.1. Let $\varepsilon^{0}$ and $\delta^{0}$ resp. $\varepsilon^{1}$ and $\delta^{1}$ be the perturbations occurring in the solution of the transfer equations (14a), (14b) with initial conditions at $t_{0}$ and $t_{1}$, respectively. Then it holds for the solution $y$ of the well-posed boundary value problem (3a)-(3c) and for the solution $\tilde{y}$ of the perturbed problem (23) $)_{0,1},(3 b),(3 c)$ that

$$
\frac{\|\tilde{y}-y\|_{\infty}}{\max \left\{1,\|\tilde{y}\|_{\infty}\right\}} \leq \kappa_{2}\left(\kappa_{3}+\kappa_{4} \kappa_{5}\right) \cdot \gamma \cdot\left(\left\|\varepsilon^{0}\right\|_{\infty}+\left\|\delta^{0}\right\|_{\infty}+\left\|\varepsilon^{1}\right\|_{\infty}+\left\|\delta^{1}\right\|_{\infty}\right),
$$

where

$$
\begin{aligned}
\mathcal{G} & \text { a generalized Green's function (cf. [15]), } \\
\kappa_{2}= & \left(t_{1}-t_{0}\right) \sup \left\{|\mathcal{G}(t, s)|: s, t \in\left[t_{0}, t_{1}\right]\right\} \\
\kappa_{3}= & \max \left\{\left|P(t) G^{-1}(t)\right|: t \in\left[t_{0}, t_{1}\right]\right\} \\
\kappa_{4}= & \max \left\{\left|Q(t) G^{-1}(t)\right|: t \in\left[t_{0}, t_{1}\right]\right\} \\
\kappa_{5}= & \max \left\{\left|P^{\prime}(t)\right|: t \in\left[t_{0}, t_{1}\right]\right\}
\end{aligned}
$$

and $\gamma$ is a constant depending only on the coefficients of equation (3a) and the boundary conditions (3b), (3c).

Before we prove this assertion, we introduce some useful estimations:

Lemma 5.2. We have

$$
\begin{aligned}
\left\|A^{T}(t) \tilde{\psi}(t)\right\|_{2} & \leq w_{0}+\varepsilon_{0}\left(t-t_{0}\right) \\
\left\|\left(\tilde{\psi}^{T}(t) A(t) A^{T}(t) \tilde{\psi}(t)\right)^{-1} \tilde{\psi}^{T}(t) A(t)\right\|_{2} & \leq \frac{w_{0}+\varepsilon_{0}\left(t-t_{0}\right)}{\frac{1}{v_{0}}-2 \varepsilon_{0} w_{0}\left(t-t_{0}\right)-\varepsilon_{0}^{2}\left(t-t_{0}\right)^{2}} \\
& \leq 3 v_{0}\left(w_{0}+\varepsilon_{0}\left(t-t_{0}\right)\right)
\end{aligned}
$$


with

$w_{0}:=\left\|A^{T}\left(t_{0}\right) \tilde{\psi}\left(t_{0}\right)\right\|_{2}, v_{0}:=\left\|\left(\tilde{\psi}^{T}\left(t_{0}\right) A\left(t_{0}\right) A^{T}\left(t_{0}\right) \tilde{\psi}\left(t_{0}\right)\right)^{-1}\right\|_{2}, \varepsilon_{0}=\sup _{t_{0} \leq t \leq t_{1}}\|\varepsilon(t)\|_{2}$.

Proof. Supposed $\varepsilon_{0}$ satisfies the inequalities $\varepsilon_{0}\left|t_{1}-t_{0}\right|<\sqrt{w_{0}^{2}+\frac{1}{v_{0}}}-w_{0}$, $2 \varepsilon_{0} w_{0}\left(t_{1}-t_{0}\right) \leq \frac{1}{3 v_{0}}$ and $\varepsilon_{0}^{2}\left(t_{1}-t_{0}\right)^{2} \leq \frac{1}{3 v_{0}}$, i.e., the perturbations are sufficiently small.

$v:=\left\|\left(\tilde{\psi}^{T} A A^{T} \tilde{\psi}\right)^{-1}\right\|_{2}$. For the initial conditions (8c) it holds that $v\left(t_{0}\right)=1$. For the definition and properties of the Dini-derivatives $D^{\star}$ the reader is referred to $[11$, p. 54].

By

$$
\left(\tilde{\psi}^{T} A A^{T} \tilde{\psi}\right)^{\prime}=\tilde{\psi}^{T} A\left(A^{T} \tilde{\psi}\right)^{\prime}+\left(\tilde{\psi}^{T} A\right)^{\prime} A^{T} \tilde{\psi} \stackrel{(19 \mathrm{a})}{=} \tilde{\psi}^{T} A \varepsilon+\varepsilon^{T} A^{T} \tilde{\psi}
$$

and

$$
\left|D^{\star}\left\|\tilde{\psi}^{T} A A^{T} \tilde{\psi}\right\|_{2}\right| \leq\left\|\left(\tilde{\psi}^{T} A A^{T} \tilde{\psi}\right)^{\prime}\right\|_{2} \leq 2\|\varepsilon\|_{2}\left\|A^{T} \tilde{\psi}\right\|_{2}
$$

it follows that

$$
\begin{gathered}
2\left\|A^{T} \tilde{\psi}\right\|_{2}\left|D^{\star}\left\|A^{T} \tilde{\psi}\right\|_{2}\right| \leq 2\|\varepsilon\|_{2}\left\|A^{T} \tilde{\psi}\right\|_{2} \\
\Rightarrow \quad\left|D^{\star}\left\|A^{T} \tilde{\psi}\right\|_{2}\right| \leq \varepsilon_{0} .
\end{gathered}
$$

On account of $w_{0}>0$ we now have

$$
\left\|A^{T}(t) \tilde{\psi}(t)\right\|_{2} \leq w_{0}+\varepsilon_{0}\left(t-t_{0}\right) .
$$

By means of the relation

$$
\left[\left(\tilde{\psi}^{T} A A^{T} \tilde{\psi}\right)^{-1}\right]^{\prime}=-\left(\tilde{\psi}^{T} A A^{T} \tilde{\psi}\right)^{-1}\left(\tilde{\psi}^{T} A A^{T} \tilde{\psi}\right)^{\prime}\left(\tilde{\psi}^{T} A A^{T} \tilde{\psi}\right)^{-1}
$$

we can derive

$$
\begin{aligned}
\left\|D^{\star} v\right\|_{2} & \leq\left\|\left[\left(\tilde{\psi}^{T} A A^{T} \tilde{\psi}\right)^{-1}\right]^{\prime}\right\|_{2} \leq 2 v^{2} \varepsilon_{0}\left(w_{0}+\varepsilon_{0}\left(t-t_{0}\right)\right) \\
\Rightarrow\left\|D^{\star}\left(\frac{1}{v}\right)\right\|_{2} & \leq 2 \varepsilon_{0}\left(w_{0}+\varepsilon_{0}\left(t-t_{0}\right)\right) .
\end{aligned}
$$

Let now $v_{0}=v\left(t_{0}\right)(>0)$, then

$$
\begin{aligned}
\frac{1}{v_{0}}-\frac{1}{v(t)} & \leq 2 \varepsilon_{0} w_{0}\left(t-t_{0}\right)+\varepsilon_{0}^{2}\left(t-t_{0}\right)^{2}, \text { i.e., } \\
v(t) & \leq \frac{1}{\frac{1}{v_{0}}-2 \varepsilon_{0} w_{0}\left(t-t_{0}\right)-\varepsilon_{0}^{2}\left(t-t_{0}\right)^{2}} .
\end{aligned}
$$


Summing up the estimations (24) and (25) provides a boundary for the perturbation term

$$
\left\|\left(\tilde{\psi}^{T}(t) A(t) A^{T}(t) \tilde{\psi}(t)\right)^{-1} \tilde{\psi}^{T}(t) A(t)\right\|_{2} \leq \frac{w_{0}+\varepsilon_{0}\left(t-t_{0}\right)}{\frac{1}{v_{0}}-2 \varepsilon_{0} w_{0}\left(t-t_{0}\right)-\varepsilon_{0}^{2}\left(t-t_{0}\right)^{2}} .
$$

This estimation is sharp because we have equality when choosing the initial values (8c) for $t=t_{0}$. The last inequality in the assertion of the lemma is now obvious. $\square$

The values for $w_{0}$ and $v_{0}$ are determined by the initial conditions of the transfer equation. The restriction of the errors $\varepsilon_{0}$ on the compact interval $\left[t_{0}, t_{1}\right]$ is plausible, too. Thus, the perturbation term is uniformly bounded. Altogether, $\tilde{B}$ and $\tilde{f}$ are regular perturbations [17] of $B$ and $f$, respectively.

Let us now prove theorem 5.1:

Proof. Let $\tilde{\psi}_{0}, \tilde{h}_{0}, \tilde{\psi}_{1}, \tilde{h}_{1}$ be the solutions of the perturbed transfer equations and let $\tilde{B}^{0}, \tilde{f}^{0}, \tilde{B}^{1}, \tilde{f}^{1}$ be the perturbed coefficients and right-hand sides, respectively, defined by the perturbations $\varepsilon^{0}, \delta^{0}, \varepsilon^{1}$ and $\delta^{1}$.

Let $y$ be the solution of $(3 \mathrm{a})$ - (3c). Then $y(t)$ has the following explicit representation $[15]$

$$
y(t)=X(t) S^{-} \hat{d}+\int_{t_{0}}^{t_{1}} \mathcal{G}(t, s) k(s) f(s) d s+Q(t) G^{-1}(t) f(t)
$$

with

$X$ is a fundamental solution of (3a),

$$
\begin{aligned}
S & =\left(\begin{array}{c}
C_{0} \\
0 \\
0
\end{array}\right)+\left(\begin{array}{c}
0 \\
C_{1} \\
0
\end{array}\right) X\left(t_{1}\right), S^{-} \text {a generalized inverse function of } S, \\
\hat{d} & =\left(\begin{array}{c}
g_{0} \\
g_{1} \\
0
\end{array}\right)-\left(\begin{array}{c}
C_{0} \\
0 \\
0
\end{array}\right) Q\left(t_{0}\right) G^{-1}\left(t_{0}\right)-\left(\begin{array}{c}
0 \\
C_{1} \\
0
\end{array}\right) Q\left(t_{1}\right) G^{-1}\left(t_{1}\right), \\
k & =\left(P+P^{\prime} Q\right) G^{-1} .
\end{aligned}
$$

$\tilde{y}(t)$ is the solution of

$$
\begin{aligned}
\tilde{\psi}_{0}(t) A(t) \tilde{y}(t) & =\tilde{h}_{0}, \\
\tilde{\psi}_{1}(t) A(t) \tilde{y}(t) & =\tilde{h}_{1}, \quad t \in\left[t_{0}, t_{1}\right], \\
Q_{s}(t) \tilde{y}(t) & =Q(t) G^{-1}(t) f(t),
\end{aligned}
$$

hence, a solution of the two initial value problems

$$
\begin{gathered}
A(t) \tilde{y}^{\prime}(t)+\tilde{B}^{0}(t) \tilde{y}(t)=\tilde{f}^{0}(t), \\
C_{0} \tilde{y}\left(t_{0}\right)=g_{0}, \\
A(t) \tilde{y}^{\prime}(t)+\tilde{B}^{1}(t) \tilde{y}(t)=\tilde{f}^{1}(t), \\
C_{1} \tilde{y}\left(t_{1}\right)=g_{1} .
\end{gathered}
$$

Trivially

$$
\tilde{B}^{0} \tilde{y}-\tilde{f}^{0}=\tilde{B}^{1} \tilde{y}-\tilde{f}^{1}
$$


holds true, consequently $\tilde{y}$ fulfils the boundary value problem

$$
\begin{aligned}
A \tilde{y}^{\prime}+B \tilde{y} & =\tilde{f}^{0}-\tilde{B}^{0} \tilde{y}+B \tilde{y} \\
C_{0} \tilde{y}\left(t_{0}\right) & =g_{0}, \\
C_{1} \tilde{y}\left(t_{1}\right) & =g_{1}
\end{aligned}
$$

i.e.,

$\tilde{y}(t)=X(t) S^{-} \hat{d}+\int_{t_{0}}^{t_{1}} \mathcal{G}(t, s) k(s)\left\{\tilde{f}^{0}(s)-\left(\tilde{B}^{0}(s)-B(s)\right) \tilde{y}(s)\right\} d s+Q(t) G^{-1}(t) f(t)$.

For sufficiently small

$$
\tilde{B}^{0}-B=A A^{T} \tilde{\psi}_{0}\left(\tilde{\psi}_{0}^{T} A A^{T} \tilde{\psi}_{0}\right)^{-1} \varepsilon^{0 T}, \quad \tilde{f}^{0}-f=A A^{T} \tilde{\psi}_{0}\left(\tilde{\psi}_{0}^{T} A A^{T} \tilde{\psi}_{0}\right)^{-1} \delta^{0}
$$

$\tilde{y}$ is thus uniquely determined.

$$
\tilde{y}(t)-y(t)=\int_{t_{0}}^{t_{1}} \mathcal{G}(t, s) k(s)\left\{\tilde{f}^{0}(s)-f(s)-\left(\tilde{B}^{0}(s)-B(s)\right) \tilde{y}(s)\right\} d s,
$$

hence,

$$
\begin{aligned}
& \frac{\|\tilde{y}(t)-y(t)\|_{\infty}}{\max \left\{1,\|\tilde{y}\|_{\infty}\right\}} \leq \kappa_{2} \cdot\left(\kappa_{3}+\kappa_{4} \cdot \kappa_{5}\right) \cdot\|A\|_{\infty} . \\
& \cdot\left(\frac{w_{0}^{0}+\varepsilon_{0}^{0}\left(t_{1}-t_{0}\right)}{\frac{1}{v_{0}^{0}}-2 \cdot \varepsilon_{0}^{0} w_{0}^{l}\left(t_{1}-t_{0}\right)-\left(\varepsilon_{0}^{0}\right)^{2}\left(t_{1}-t_{0}\right)^{2}}+\right. \\
&\left.+\frac{w_{0}^{1}+\varepsilon_{0}^{1}\left(t_{1}-t_{0}\right)}{\frac{1}{v_{0}^{1}}-2 \cdot \varepsilon_{0}^{1} w_{0}^{1}\left(t_{1}-t_{0}\right)-\left(\varepsilon_{0}^{1}\right)^{2}\left(t_{1}-t_{0}\right)^{2}}\right) . \\
& \cdot\left(\left\|\varepsilon^{0}\right\|_{\infty}+\left\|\delta^{0}\right\|_{\infty}+\left\|\varepsilon^{1}\right\|_{\infty}+\left\|\delta^{1}\right\|_{\infty}\right) .
\end{aligned}
$$

By $\gamma:=\|A\|_{\infty} \cdot\left(3 v_{0}^{0}\left(w_{0}^{0}+\varepsilon_{0}^{0}\left(t_{1}-t_{0}\right)\right)+3 v_{0}^{1}\left(w_{0}^{1}+\varepsilon_{0}^{1}\left(t_{1}-t_{0}\right)\right)\right)$ the assertion is proved. $\mathrm{C}$

Comparing the original differential-algebraic equation (3a) with the perturbed one (23) we notice that the relevant subspaces, i.e., the nullspaces of the leading coefficient matrix as well as the solution spaces of the homogeneous equations are identical. We have

$$
\begin{aligned}
\tilde{G}^{-1}=(A+\tilde{B} Q)^{-1} & =\left(G+A A^{T} \tilde{\psi}\left(\tilde{\psi}^{T} A A^{T} \tilde{\psi}\right)^{-1} \varepsilon^{T} Q\right)^{-1} \\
& =\left(I-P A^{T} \tilde{\psi}\left(\tilde{\psi}^{T} A A^{T} \tilde{\psi}\right)^{-1} \varepsilon^{T} Q\right) G^{-1}
\end{aligned}
$$

If we consider additionally the state variable systems [10, Theorem 1.2.14] of the index 1 DAE (3a)

$$
\begin{aligned}
u^{\prime} & =\left(P^{\prime} P_{s}-P G^{-1} B\right) u+P\left(I+P^{\prime}\right) G^{-1} f, \\
y & =P_{s} u+Q G^{-1} f .
\end{aligned}
$$


and

$$
\begin{aligned}
\tilde{u}^{\prime} & =\left(P^{\prime} P_{s}-P \tilde{G}^{-1} \tilde{B}\right) \tilde{u}+P\left(I+P^{\prime}\right) \tilde{G}^{-1} \tilde{f}, \\
\tilde{y} & =P_{s} \tilde{u}+Q G^{-1} f
\end{aligned}
$$

of (23), then we see immediately that (27) represents a regular perturbation of (26). Therefore, we speak of regularly perturbed differential-algebraic equations if the basic structure is invariant and only the coefficients and right-hands sides in the basic parts are subject to smaller perturbations.

Hence, we notice that the transfer of the boundary conditions (14a), (14b) is stable in the sense that small perturbations of the right-hand side in the transfer equations represent a regular perturbation of the given index 1 equation of the same size.

6. Numerical Examples. The algorithm used for the transfer of the boundary conditions for the numerical examples reads:

1. Solve the equations (14a), (14b) with the corresponding initial conditions $(8 \mathrm{a}),(8 \mathrm{~b})$ or $(8 \mathrm{c}),(8 \mathrm{~d})$ on the left resp. right boundary of the interval, store the solution values.

2. Solve the algebraic equations

$$
\begin{aligned}
\psi_{i}^{T}(t) A(t) y(t) & =h_{i}(t), & i=0,1, \\
Q_{s}(t) y(t) & =Q(t) G^{-1}(t) f(t), & t \in\left[t_{0}, t_{1}\right],
\end{aligned}
$$

wherever the solution is needed.

In order to solve the examples numerically, the code TRANSFER developed by the author has been used. This code organizes the integration of the transfer equations in the corresponding directions and, after having solved the algebraic equations, it returns the results to the main program at the desired moments. The transfer equations were developed with the aid of programs for the linear algebra from the NAG-library. For the integration of the transfer equation the code DASSL [18], [7] was used. The tolerance for DASSL was chosen as atol $=r t o l=1 D-6$. The solution intervals were subdivided equidistantly and values computed by DASSL were given at the grid points. The linear system of equations (17a), (17b) was solved with an accuracy of $1 D-8$ at each grid point. The program PQ [14] was used for computing the projectors.

6.1. Example of an Ordinary Differential Equation. We start with a regular differential equation to show the advantage of the Abramov transfer to those readers who have not been familiar with this transfer by now. The problem [4, p. 121, Example 3.13, p. 169, Example 4.13] reads

$$
y^{\prime}-\left(\begin{array}{cc}
-\lambda \cos (2 \omega t) & \omega+\lambda \sin (2 \omega t) \\
-\omega+\lambda \sin (2 \omega t) & \lambda \cos (2 \omega t)
\end{array}\right) y=0, \quad 0<t<\Pi .
$$

A fundamental matrix for this differential equation reads

$$
Y(t)=\left(\begin{array}{cc}
\cos (\omega t) & \sin (\omega t) \\
-\sin (\omega t) & \cos (\omega t)
\end{array}\right)\left(\begin{array}{cc}
e^{-\lambda t} & 0 \\
0 & e^{\lambda t}
\end{array}\right) .
$$

This representation reveals the dichotomy of the solution space immediately [9], [20]. For different $\omega$ the solutions rotate with different speed. The eigenvalues of the matrix 
in equation (29) are $\pm \sqrt{\lambda^{2}-\omega^{2}}$, where $\pm \lambda$ represent the kinematic eigenvalues [4]. For increasing $\omega$ the eigenvalues drift away from the kinematic eigenvalues, for $\omega>\lambda$ they become imaginary and do not contain any information about the dichotomy, which does not change with $\omega$.

A general solution of the problem is given by

$$
y(t)=\left(\begin{array}{cc}
\cos (\omega t) & \sin (\omega t) \\
-\sin (\omega t) & \cos (\omega t)
\end{array}\right)\left(\begin{array}{cc}
e^{-\lambda t} & 0 \\
0 & e^{\lambda t}
\end{array}\right)\left(\begin{array}{c}
\alpha \\
\beta
\end{array}\right),
$$

$\alpha, \beta \in \mathbb{R}$. The solution has been computed in the interval $[0.001 ; \Pi-0.001]$ for $\alpha=1, \beta=1$.

In order to formulate the boundary conditions, the matrices

$$
C_{0}=\left(\cos \left(\omega t_{0}\right),-\sin \left(\omega t_{0}\right)\right), \quad C_{1}=\left(\sin \left(\omega t_{1}\right), \cos \left(\omega t_{1}\right)\right)
$$

have been used. For $\omega=0$ and $\lambda \geq 0$ the problem is well-conditioned then because the decaying parts of the fundamental solution are controlled by conditions on the left boundary of the interval and the growing parts by conditions on the right boundary of the interval. The authors of [4] emphasize that the Riccati-method does not work well for $\lambda=1$ and greater $\omega$, whereas it works well for $\omega=1$ and greater $\lambda$.

For various pairs of values of $\lambda$ and $\omega$ the exact solution is of the following form:

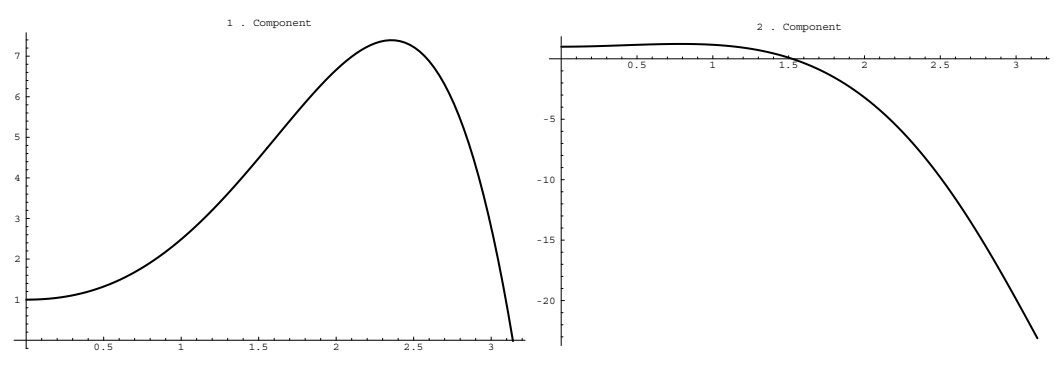

$$
\lambda=1, \omega=1
$$

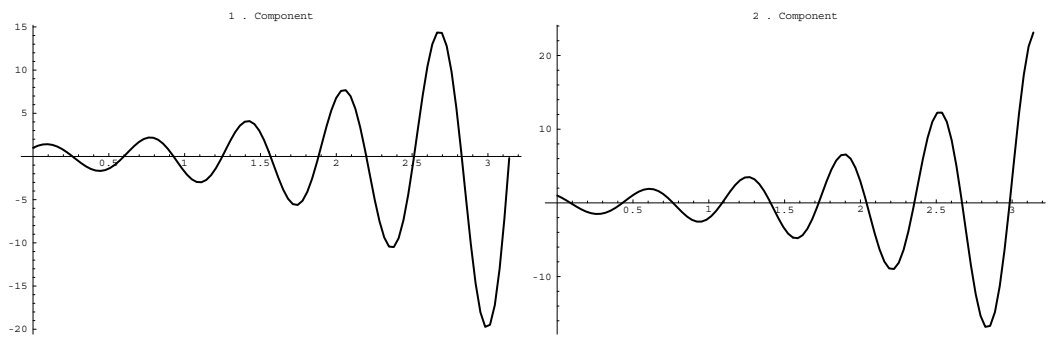

$\lambda=1, \omega=10$ 


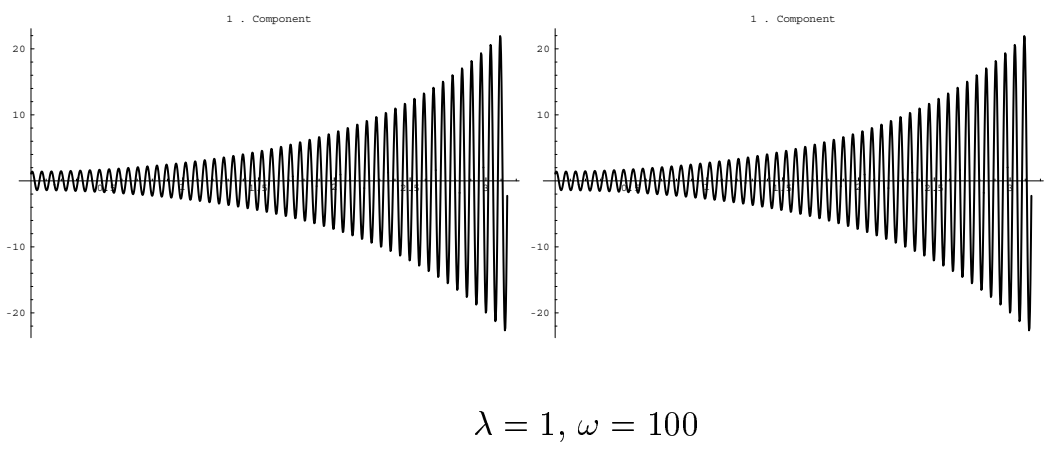

In the first choice of the initial conditions (8a), (8b) we have considered the relative errors

$$
\text { error }=\max _{i} \frac{\left\|\tilde{y}\left(t_{i}\right)-y\left(t_{i}\right)\right\|_{2}}{\left\|y\left(t_{i}\right)\right\|_{2}}
$$

where $y$ denotes the exact solution and $\tilde{y}$ the numerical one. For the initial values (8c), (8d) the absolute errors have been computed. These errors were of the same magnitude as the relative errors in case of the other choice of the initial value such that we can do here without the exact numbers.

The relative errors in the computation of the solution in the transfer are shown in the following table:

\begin{tabular}{|c||c|c|c|c|c|c|}
\hline \multicolumn{1}{|c||}{$\omega$} & 1 & 10 & 20 & 100 & 200 & 1000 \\
\hline \hline 1 & $8.1 \mathrm{D}-06$ & $1.5 \mathrm{D}-05$ & $2.5 \mathrm{D}-05$ & $7.7 \mathrm{D}-04$ & $1.3 \mathrm{D}-03$ & $1.4 \mathrm{D}-02$ \\
\hline 2 & $8.3 \mathrm{D}-06$ & $2.3 \mathrm{D}-05$ & $1.3 \mathrm{D}-04$ & $7.5 \mathrm{D}-04$ & $6.2 \mathrm{D}-03$ & $1.7 \mathrm{D}-02$ \\
\hline 5 & $3.2 \mathrm{D}-04$ & $1.1 \mathrm{D}-05$ & $1.3 \mathrm{D}-04$ & $7.3 \mathrm{D}-04$ & $5.1 \mathrm{D}-03$ & $1.6 \mathrm{D}-02$ \\
\hline 10 & $3.4 \mathrm{D}-04$ & $3.8 \mathrm{D}-05$ & $3.2 \mathrm{D}-05$ & $7.1 \mathrm{D}-04$ & $2.7 \mathrm{D}-03$ & $1.5 \mathrm{D}-02$ \\
\hline 100 & $8.2 \mathrm{D}-04$ & $8.9 \mathrm{D}-04$ & $8.9 \mathrm{D}-04$ & $2.6 \mathrm{D}-04$ & $4.5 \mathrm{D}-04$ & $1.7 \mathrm{D}-03$ \\
\hline 200 & $1.4 \mathrm{D}-03$ & $1.4 \mathrm{D}-03$ & $1.5 \mathrm{D}-03$ & $1.6 \mathrm{D}-03$ & $4.2 \mathrm{D}-03$ & $1.2 \mathrm{D}-03$ \\
\hline
\end{tabular}

The transfer method provides good results for great $\omega$ as well as for the stiff case $\lambda=200$.

6.2. An Index-1 Example. The following example has been constructed as a numerical example of a linear index 1 equation:

By means of the matrices $E$ and $F$

$$
E=\left(\begin{array}{ccccc}
1 & 2 & 3 & 4 & 5 \\
1 & 4 & 9 & 16 & 25 \\
1 & 8 & 27 & 64 & 125 \\
1 & 16 & 81 & 256 & 625 \\
1 & 32 & 243 & 1024 & 3125
\end{array}\right), \quad F=\left(\begin{array}{ccccc}
-1 & -2 & 1 & 2 & 3 \\
1 & 4 & 1 & 4 & 9 \\
-1 & -8 & 1 & 8 & 27 \\
1 & 16 & 1 & 16 & 81 \\
-1 & -32 & 1 & 32 & 243
\end{array}\right)
$$

we can construct $A$ and $B$ as follows:

$$
A=E \cdot \operatorname{diag}(1,1,1,0,0) \cdot F^{-1}, B=E \cdot \operatorname{diag}\left(0,0, t+1, t+2,(t+1)^{2}\right) \cdot F^{-1} .
$$


The solution $y$ is given by

$$
y(t)=\left(\begin{array}{c}
\sin (t)+\cos (5 t) \\
t^{2}+3 \\
e^{-t} \\
\cos (t) \cdot e^{t} \\
\frac{1}{t+1}
\end{array}\right) .
$$

The right-hand side of the problem $q$ is computed by inserting $A, B, y$ and $y^{\prime}$

$$
A y^{\prime}+B y=q .
$$

This example is tractable with index 1 . The equations

$$
\left(\begin{array}{ccccc}
\frac{7}{5} & \frac{1}{12} & -\frac{11}{24} & -\frac{1}{12} & \frac{7}{120} \\
\frac{7}{10} & \frac{7}{6} & -\frac{19}{24} & -\frac{1}{6} & \frac{11}{120}
\end{array}\right) \cdot y(0)=\left(\begin{array}{c}
\frac{7}{6} \\
\frac{10}{3}
\end{array}\right)
$$

and

$$
\begin{aligned}
\left(\begin{array}{lllll}
-\frac{1}{10} & \frac{4}{3} & \frac{19}{24} & -\frac{4}{3} & \frac{37}{120}
\end{array}\right) \cdot y(1)= \\
\\
\quad \frac{439}{80}+\frac{19}{24} \cdot e^{-1}-\frac{4}{3} e \cos (1)-\frac{1}{10}(\cos (5)+\sin (1))
\end{aligned}
$$

have been used as boundary conditions.

First, we have considered the results of the computation when choosing the initial conditions according to the equations (8a), (8b). The relative error over all five components in the interval $[0,1]$ is $2.035 D-05$.

The variation of the product $\psi^{T} A A^{T} \psi$ was also investigated. At the starting time $t=0(1)$ a reference matrix was computed and this matrix was used for a comparison with the above matrix at the actual time. Again, the relative error was considered. The maximum of relative errors on the solution interval of the - according to theory - constant matrix was $1.828 D-6$.

When choosing the initial conditions according to the equations $(8 \mathrm{c}),(8 \mathrm{~d})$ we had the maximal error over all five components of the solution on the interval $7.676 D-5$.

The product $\psi^{T} A A^{T} \psi$ was here approximately the unit matrix, the maximal deviations were of the size of the relative errors for the other choice of the initial values.

In the further test examples we obtained similarly good results as those presented above.

REMARK 6.1. The restriction $C_{i} P\left(t_{i}\right)=C_{i}$ is due to the choice of the initial values for the adjoint equations (4), (5)

$$
\phi\left(t_{i}\right)=P_{\star s}\left(t_{i}\right) \cdot A^{T \dagger}\left(t_{i}\right) \cdot C_{i}^{T}\left(\cdot L_{i}\right) .
$$

For the considered form of the transfer equations this restriction is essential, as the following simple example will show:

$$
\left(\begin{array}{ll}
1 & 0 \\
0 & 0
\end{array}\right) y^{\prime}+\left(\begin{array}{ll}
0 & 0 \\
0 & 1
\end{array}\right) y=\left(\begin{array}{l}
0 \\
1
\end{array}\right), \quad y_{1}(0)-y_{2}(0)=0
$$

The unique solution reads $y_{0} \equiv y_{1} \equiv 1$. The transfer equations are simplified to

$$
\begin{aligned}
\psi_{1}^{\prime} & =0 \\
\psi_{2} & =0 \\
h^{\prime}-\psi_{2} & =0 .
\end{aligned}
$$


With the initial values (8a), (8b)

$$
\begin{aligned}
& \psi(0)=P_{\star s}(0) \cdot A^{T \dagger}(0) \cdot C_{0}^{T}=\left(\begin{array}{ll}
1 & 0 \\
0 & 0
\end{array}\right) \cdot\left(\begin{array}{ll}
1 & 0 \\
0 & 0
\end{array}\right) \cdot\left(\begin{array}{c}
1 \\
-1
\end{array}\right)=\left(\begin{array}{l}
1 \\
0
\end{array}\right), \\
& h(0)=0
\end{aligned}
$$

we obtain $\psi_{1} \equiv 1, \psi_{2} \equiv h \equiv 0$, which results in $y_{1} \equiv 0$ and $y_{2} \equiv 1$, however.

If the boundary conditions are only established for the P-components $y_{1}(0)=1$, the transfer provides the correct results.

\section{Acknowledgement}

I am grateful to the referees for the careful reading of the manuscript and the many hints that helped to improve it.

\section{REFERENCES}

[1] A. Aвramov, On the transfer of boundary conditions for systems of ordinary linear differential equations (a variant of the dispersive method) (in Russian), Zh. Vychisl. Mat. Mat. Fiz., 1(3) (1961), pp. 542-545.

[2] — - Vorlesung: Einige Methoden zur Lösung von Randwertaufgaben für Systeme linearer gewöhnlicher Differentialgleichungen. Gehalten an der Humboldt-Universität zu Berlin, Wintersemester 1992/93.

[3] A. Abramov, E. Birger, N. Konyukhova, and V. Ulyanova, On methods of numerical solution of boundary value problems for systems of linear ordinary differential equations, in Colloquia Mathematica Societatis János Bolyai 22. Numerical Methods ed.: Rózsa, North-Holland, Keszthely (Hungary), 1977, pp. 33-67.

[4] U. Ascher, R. MattheiJ, And R. Russel, Numerical Solution of boundary value problems for ordinary differential equations, Prentice Hall Series in Computational Mathematics, Prentice Hall, Englewood Cliffs, New Jersey, 1988.

[5] K. BALla, Linear subspaces for linear DAEs of index 1, Computers Math. Applic., 31 (1996), pp. 81-86.

[6] K. BAlla AND R. MÄRz, Transfer of boundary conditions for DAEs of index 1, SIAM J. Numer. Anal., 33 (1996).

[7] K. Brenan, S. Campbell, and L. Petzold, Numerical solution of initial-value problems in ordinary differential-algebraic equations, North Holland Publishing Co., New York, 1989.

[8] G. J. COOPER, Stability of Runge-Kutta methods for trajectory problems, IMA J. Numer. Anal., 7 (1987), pp. 1-13.

[9] W. Coppel, Dichotomies in stability theory, Lecture Notes in Mathematics, Springer Verlag, Berlin-Heidelberg-New York, 1978.

[10] E. Griepentrog and R. März, Differential-algebraic equations and their numerical treatment, Teubner-Texte zur Mathematik; 88, Teubner, Leipzig, 1986.

[11] E. Hairer, S. P. NøRSETt, AND G. WANner, Solving ordinary differential equations I, Springer Series in Computational Mathematics, Springer-Verlag, Berlin, Heidelberg, New York, 1987.

[12] E. Kamke, Differentialgleichungen. Lösungsmethoden und Lösungen. I. Gewöhnliche Differentialgleichungen, Leipzig, Akad.-Verl., 1959.

[13] R. Lagrange, Sur les systèmes adjoints d'Équations différentielles linéaires, Acta Math., 48 (1926), pp. 179-201.

[14] R. Lamour, Personal communication. 1994-96.

[15] M. LENTINI AND R. MÄRZ, Conditioning and dichotomy in differential algebraic equations, SIAM J. Numer. Anal., 27 (1990), pp. 1519-1526.

[16] R. M̈̈RZ, Numerical methods for differential-algebraic equations, Acta Numerica, (1992), pp. 141-198.

[17] R. E. O'MALley, Singular perturbation methods for ordinary differential equations, vol. 89 of Applied mathematical sciences, Springer-Verlag, New York, 1991.

[18] L. Petzold, A description of DASSL: A differential/algebraic system solver, in Proc. 10th IMACS World Congress, August 8-13 Montreal 1982, 1982.

[19] J. Stoer, Numerische Mathematik I, Heidelberger Taschenbücher; 105, Springer-Verlag, Berlin, 5th ed., 1989. 
[20] P. M. van Loon, Continuous Decoupling Transformations for Linear Boundary Value Problems, PhD thesis, Technische Universiteit Eindhoven, Eindhoven, 1987. 